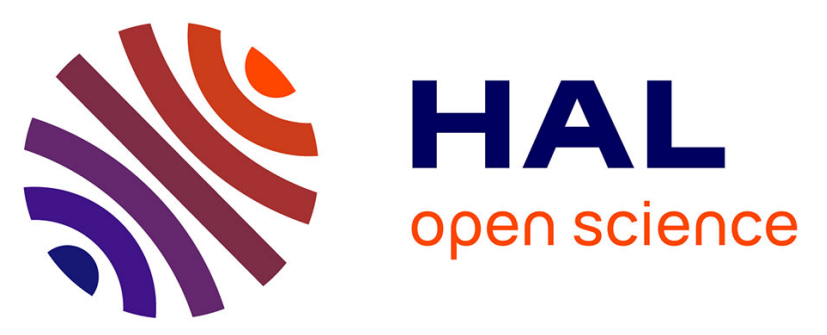

\title{
AMÉLIORATION DE L'INTELLIGIBILITÉ DANS DES LOCAUX ACOUSTIQUEMENT DÉFAVORABLES PAR COMPRESSION/EXPANSION
}

J. Maisano, M. Rossi, T. Styger

\section{- To cite this version:}

J. Maisano, M. Rossi, T. Styger. AMÉLIORATION DE L'INTELLIGIBILITÉ DANS DES LOCAUX ACOUSTIQUEMENT DÉFAVORABLES PAR COMPRESSION/EXPANSION. Journal de Physique IV Proceedings, 1992, 02 (C1), pp.C1-331-C1-334. 10.1051/jp4:1992171 . jpa-00251243

HAL Id: jpa-00251243

https://hal.science/jpa-00251243

Submitted on 1 Jan 1992

HAL is a multi-disciplinary open access archive for the deposit and dissemination of scientific research documents, whether they are published or not. The documents may come from teaching and research institutions in France or abroad, or from public or private research centers.
L'archive ouverte pluridisciplinaire HAL, est destinée au dépôt et à la diffusion de documents scientifiques de niveau recherche, publiés ou non, émanant des établissements d'enseignement et de recherche français ou étrangers, des laboratoires publics ou privés. 


\title{
AMÉLIORATION DE LINTELLIGIBILITE DANS DES LOCAUX ACOUSTIQUEMENT DEFAVORABLES PAR COMPRESSION/EXPANSION
}

\author{
J. MAISANO, M. ROSSI et T. STYGER \\ Laboratoire d'Electromagnétisme et d'Acoustique, Département d'Electricité, Ecole Polytechnique \\ Fédérale de Lausanne, CH-1015 Lausanne, Switzerland
}

\begin{abstract}
This paper presents a speech compression/expansion method that aims to improve the intelligibility in highly reverberant industrial premises where acoustic corrections are excluded. By placing a digital signal processor upstream of the sound system, it is possible, using frequency-based criteria, to detect in real time the phonemes requiring compression (typically the vowels) and those requiring expansion (for example the occlusive consonants). The assessment of subjective lntelligibility carried out on a workstation shows an improvement in the CVC scores in the range of $20 \%$.
\end{abstract}

\section{Introduction}

Certains locaux publics ou industriels, par exemple dans les centrales de production d'énergie, présentent une acoustique très défavorable pour la parole, alors que leur fonction requiert la transmission de messages, par exemple ordres, consignes de sécurité ou alarmes, via un système électroacoustique de diffusion. De tels locaux ont des temps de réverbération élevés (plusieurs secondes, surtout aux fréquences basses, moins aux fréquences élevées) et très souvent, pour des impératifs de sécurité ou autres, une correction acoustique est exclue ou lorsqu'elle est possible, n'est cependant pas suffisante pour garantir l'intelligibilité exigée, malgré une disposition appropriée des haut-parleurs.

L'effet de la réverbération sur la parole est bien connu: dégradation de l'intelligibilité par chevauchement temporel des phonèmes, surtout des voyelles, de niveaux relativement élevés aux fréquences basses déjà, sur les consonnes, de niveaux moindres et dont le spectre s'étale vers les fréquences élevées. Améliorer l'intelligibilité en présence de réverbération, c'est réduire ce chevauchement. C'est avec cet objectif que nous avons entrepris l'étude de la faisabilité d'un conditionnemeent de la parole en amont du système électroacoustique de diffusion. Le problème avait déjà fait l'objet d'une expérimentation par des techniques analogiques il y a une dizaine d'années. Les tests d'intelligibilité, effectués dans une cathédrale avec des sujets naïfs, avaient montré l'intérêt d'une compression/expansion par bande, mais le peu de souplesse des équipements alors disponibles ne permettait pas l'adaptation à l'acoustique particulière d'un espace donné. L'apparition des processeurs numériques de signaux DSP nous a conduit à reprendre le principe de base, à le perfectionner et à l'implanter de manière plus performante. 


\section{Robustesse des phonèmes}

Le premier problème a été de classer les phonèmes en catégories selon leur robustesse à la réverbération. Cette classification a été établie sur la base de la dégradation des phonèmes dans une salle réverbérante, présentant des temps de réverbération de $20 \mathrm{~s} \mathrm{à} 125 \mathrm{~Hz}$ et de $5 \mathrm{~s}$ à $5 \mathrm{kHz}$, avec des locuteurs masculins, et en tenant compte de la coarticulation. Le tableau ci-après donne les résultats en terme de robustesse forte $F$, moyenne $M$ et faible $L$. Il faut noter que cette classification est relativement grossière et n'indique qu'une tendance générale dans le contexte vocalique le plus fréquent. Selon ce contexte, certains phonèmes changent de catégories (indication entre parenthèses dans le tableau).

\begin{tabular}{|l|c|c|c|}
\hline Robustesse & forte F & moyenne M & faible L \\
\hline Voyelles orales & $\bullet$ & $\bullet$ & \\
\hline Voyelles nasales & & $\bullet$ & $(\cdot)$ \\
\hline Cons. occlusives & & $(\bullet)$ & \\
\hline Cons. fricatives & $(\bullet)$ & $(\bullet)$ & $(\cdot)$ \\
\hline Cons. liquides & & $(\cdot)$ & \\
\hline Semi-voyelles & & $\bullet$ & \\
\hline
\end{tabular}

\section{Identification des catégories}

En vue des conditionnements envisagés, il faut identifier en temps réel l'appartenance du phonème courant à l'une des 3 catégories. On a cherché pour cela à mettre en oeuvre des critères fréquentiels, aussi pertinents que possible, mais surtout pouvant s'implanter simplement sous forme d'un banc de filtres suivis de détecteurs de niveau.

Par expérimentation, on a d'abord déterminé les bandes de fréquence contenant des indices caractéristiques des différents phonèmes, dans l'idée de déterminer un découpage fréquentiel susceptible de permettre la discrimination, même sommaire des catégories.

Finalement, on a abouti aux bandes suivantes, avec le schéma brut donné des indices acoustiques par catégorie:

\begin{tabular}{|l|l|l|l|}
\hline & \multicolumn{3}{|c|}{ CATEGORIE } \\
\hline BANDE Hz & \multicolumn{1}{|c|}{ F } & \multicolumn{1}{|c|}{ M } \\
\hline 1:80 a 400 & & $\begin{array}{l}\text { Premier formant } \\
\text { des voyelles } \\
\text { Barres de voisement }\end{array}$ & Barres de voisement \\
\hline 2: 400 à 800 & $\begin{array}{l}\text { Premier formant } \\
\text { des voyelles } \\
\text { des voyelles nasales }\end{array}$ & Formant du /R/ \\
\hline 3: 800 à 1200 & & $\begin{array}{l}\text { Premier formant } \\
\text { des voyelles nasales }\end{array}$ & \\
\hline 4: 1200 à 2500 & & & $\begin{array}{l}\text { Barres d'explosion } \\
\text { des occlusives } \\
\text { Bruit du } / \mathbb{R} /\end{array}$ \\
\hline 5: 2500 à 5000 & $\begin{array}{l}\text { Maxima d'amplitude } \\
\text { du bruit des } \\
\text { fricatives }\end{array}$ & $\begin{array}{l}\text { Barres d'explosion } \\
\text { des occlusives }\end{array}$ \\
\hline
\end{tabular}


L'analyse des durées relatives des phonèmes, même si elle a montré des différences significatives, n'a cependant pas permis de dégager des critères de différenciation à la fois simples et pertinents, applicables en parole continue.

\section{Implantation}

Le principe de base est d'effectuer une compression des phonèmes de la catégorie $F$ et une expansion de ceux de la L, ceux de la $M$ ne subissant aucune action. Ces conditionnements sont à réaliser par un processeur de signal DSP 56000. La puissance de calcul limitée d'une seule carte DSP oblige à n'envisager le conditionnement que des phonèmes les plus fréquents de la langue française. Aussi, n'a-t-on cherché qu'à optimiser le traitement:

- des voyelles orales et nasales

- des consonnes liquides $/ \mathrm{R} /$ et $/ \mathrm{I} /$ et nasales $/ \mathrm{m} /$ et $/ \mathrm{n} /$

- des occlusives sonores et sourdes, excepté / $/$ / et $/ g /$

- des fricatives $/ \mathrm{s} / \mathrm{et} / \mathrm{v} /$

Pour le développement du processus, la carte DSP a été installée dans un ordinateur personnel PC compatible, un interface utilisateur graphique permettant la visualisation et le pilotage par souris des différentes fonctions du DSP. La figure ci-après reproduit l'écran du PC pour la configuration la plus simple des gains et caractéristiques de compression/expansion: on y reconnaît un banc de filtres ( 5 voies, chacune constituée de filtres de Linkwitz passe-haut et passe-bas du 2ème ordre en cascade), des compresseurs/expanseurs dans les voies 1 et 5 (avec valeurs du seuil et des gains en-dessus et en-dessous), les voies 2 à 4 ne comprenant qu'un gain (de -30 à $+30 \mathrm{~dB}$ ). La fréquence d'échantillonnage est de $12 \mathrm{kHz}$. Il est possible de supprimer tout conditionnement par le bouton "Bypass" ou l'une ou l'autre voie par les boutons "Cut".

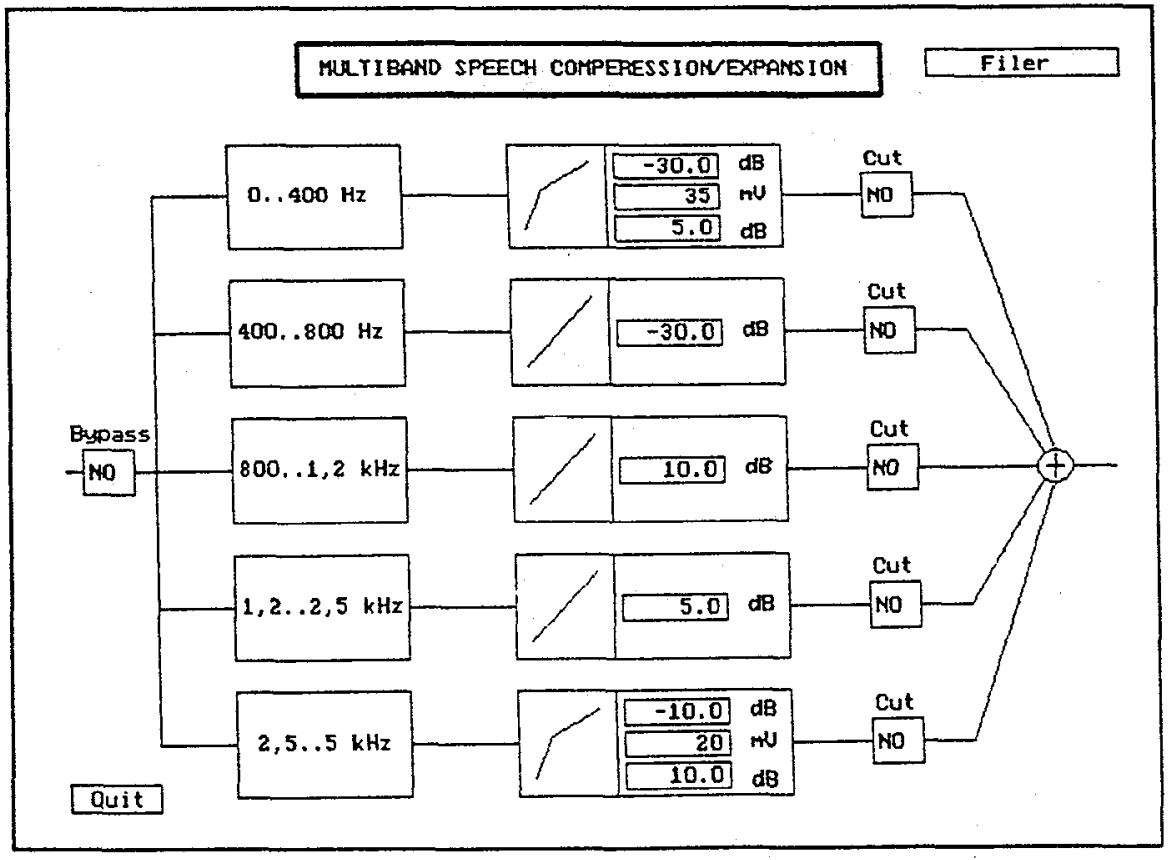




\section{Expérimentation}

La souplesse de programmation du système a permis d'expérimenter les améliorations d'intelligibilité pour différents réglages de la configuration la plus simple, sous les conditions de réverberation de la salle réverbérante citée sous 2 . Les tests d'intelligibilité ont été réalisés à l'aide de la station SESI [1] avec 16 listes de 10 logatomes. Les résultats sont significatifs à $90 \%$ pour un écart de score de $5 \%$. Pour deux réglages jugés subjectivement très bons, les résultats sont les suivants:

\begin{tabular}{|c|c|c|}
\hline Réglage & Score \% & Amélioration \% \\
\hline Bypass & 55 & 24 \\
\hline 8 & 79 & 20 \\
\hline 13 & 75 & 24 \\
\hline
\end{tabular}

Les différentes configurations et réglages se distinguen non-seulement par leurs scores, mais aussi par les modifications du naturel de la voix plus ou moins agréables ou confortables. Ainsi, le réglage 13, un peu moins efficace que le 8 , est jugé beaucoup plus acceptable que ce dernier, car altérant moins le timbre.

\section{Poursuite des travaux}

Les actions suivantes vont suivre

- analyse des expérimentations par les matrices de confusion

- améliorations des critères de différenciation des phonèmes

- adjonction d'une unité de décision intégrant les indices acoustiques détectés dans les différentes voies

- évaluation systématique dans différents locaux et avec différents locuteurs masculins et féminins

\section{Bibliographie}

[1] F. Kehlstadt, L. Morellini, "Tests semi-automatiques de l'intelligibilité de la parole dans les systèmes de transmission", Communications AGEN, No 52, novembre 1990, pp. 53-57 